\title{
Donor-specific B Cell Memory in Alloimmunized Kidney Transplant Recipients: First Clinical Application of a Novel Method
}

\author{
Caroline Wehmeier, MD, ${ }^{1}$ Gonca E. Karahan, PhD, ${ }^{1}$ Juliette Krop, BSc, ${ }^{1}$ Yvonne de Vaal, BSc, ${ }^{1}$
} Janneke Langerak-Langerak, BSc, ${ }^{1}$ Isabelle Binet, MD, ${ }^{2}$ Stefan Schaub, MD, ${ }^{3}$ Dave L. Roelen, PhD, ${ }^{1}$ Frans H.J. Claas, PhD, ${ }^{1}$ Sebastiaan Heidt, PhD, ${ }^{1}$ and the Swiss Transplant Cohort Study*

\begin{abstract}
Background. HLA-specific memory B cells may contribute to the serum HLA antibody pool upon antigen reexposure. The aim of this pilot study was to investigate the presence of concurrent donor-specific memory B cell-derived HLA antibodies (DSA-M) in renal allograft recipients with pretransplant donor-specific HLA antibodies (DSA) and its association with occurrence of antibody-mediated rejection (AMR) using a recently developed method. Methods. Twenty patients with Luminex single antigen bead (SAB) assay-defined DSA but negative complement-dependent cytotoxicity crossmatches were enrolled. Plasma samples and peripheral blood mononuclear cells were collected at 3 timepoints (pretransplant, mo 6 , mo 12). We analyzed IgG-purified and concentrated culture supernatants from polyclonally activated peripheral blood mononuclear cells using SAB assays and compared HLA antibody profiles with same day plasma results. Results. Plasma SAB analysis revealed 35 DSA in 20 patients pretransplant. DSA-M were detected in 9 of 20 (45\%) patients and for 10 of 35 specificities (29\%). While median mean fluorescence intensity values of DSA with concurrent DSA-M (5877) were higher than those of DSA without DSA-M (1476), 3 of 6 patients with AMR and low mean fluorescence intensity DSA $(<3000)$ had DSA-M. Overall, pretransplant DSA/DSA-M $M_{\text {pos }}$ allograft recipients showed a higher incidence of biopsy-proven (sub)clinical $\operatorname{AMR}(P=0.032)$ and a higher extent $(g \geq 1+$ ptc $\geq 1)$ of microvascular inflammation $(67 \%$ vs $9 \%, P=0.02)$. In 17 patients (28 DSA) with posttransplant analyses, persisting DSA posttransplant had more often DSA-M (6/12; 50\%) than nonpersisting DSA $(2 / 16 ; 13 \%)$. Conclusions. Assessment of DSA-M might be a novel tool to supplement serum HLA antibody analysis for pretransplant risk stratification in patients with DSA.
\end{abstract}

(Transplantation 2020;104: 1026-1032).

Received 2 February 2019. Revision received 23 July 2019.

Accepted 24 July 2019

${ }^{1}$ Department of Immunohematology and Blood Transfusion, Leiden University Medical Center, Leiden, the Netherlands.

${ }^{2}$ Department of Nephrology and Transplantation Medicine, Kantonsspital St. Gallen, St. Gallen, Switzerland.

${ }^{3}$ Clinic for Transplantation Immunology and Nephrology, University Hospital Basel, Basel, Switzerland.

*The members of the STCS are indicated in the Acknowledgments.

C.W. designed and performed the research, analyzed the data, and wrote the article. G.E.K. analyzed the data and wrote the article. J.K., Y.d.V., and J.LL. performed the research and analyzed the data. I.B. participated in study design. S.S. and F.H.J.C. participated in study design and wrote the paper. D.R. assisted in data acquisition and wrote the paper. S.H. designed the research, analyzed the data, and wrote the article.

The authors declare no conflicts of interest.

This work was supported by the Swiss National Science Foundation (SNF), the Gottfried and Julia Bangerter-Rhyner Foundation (Switzerland), and the National Reference Center for Histocompatibility Testing (Leiden, the Netherlands).

Supplemental digital content (SDC) is available for this article. Direct URL citations appear in the printed text, and links to the digital files are provided in the HTML text of this article on the journal's Web site (www.transplantjournal.com).

Correspondence: Sebastiaan Heidt, PhD, Department of Immunohematology and Blood Transfusion, Leiden University Medical Center, Albinusdreef 2, 2333 ZA Leiden, the Netherlands. (s.heidt@/umc.nl).

Copyright $\odot 2019$ Wolters Kluwer Health, Inc. All rights reserved.

\section{INTRODUCTION}

In kidney transplantation, current immunological pretransplant risk assessment is based on detection of donorspecific HLA antibodies (DSA) in the patients' serum. Their presence is a major risk factor for antibody-mediated rejection (AMR) and inferior allograft outcomes. ${ }^{1,2}$ In the era of highly sensitive Luminex single antigen bead (SAB) technology, transplantation in the absence of DSA is not always possible. For DSA-positive patients, options to transplant include desensitization as well as treatment with an intensified induction immunosuppression. ${ }^{3-5}$ However, the posttransplant course of patients transplanted across the DSA barrier is highly variable and cannot be sufficiently predicted by antibody properties, such as antibody strength, composition of IgG subclasses, and complementbinding capacities. ${ }^{6,7}$

Previous studies showed that persisting or increasing levels of HLA antibodies after transplantation are associated with a higher risk for AMR. ${ }^{8-10}$ Rebound and/or persistence of DSA posttransplant may be derived from dormant circulating memory $\mathrm{B}$ cells that differentiate into antibody-producing cells upon antigen reencounter or bystander activation. ${ }^{11-13}$

So far, assessment of the peripheral HLA-specific memory B cell pool for risk stratification has been either labor-intensive or lacked sensitivity due to low $\operatorname{IgG}$ concentrations and possibly IgM interference in B cell 
supernatants following in vitro stimulation. ${ }^{14-17}$ Our group recently presented an easy-to-perform technique for sensitive detection of HLA antibodies derived from circulating memory B cells. ${ }^{18}$ For this, IgG of supernatants from polyclonally activated memory B cells is isolated, concentrated, and analyzed using SAB technology, enabling direct comparison with serum HLA antibody profiles.

In this pilot study, the method was applied to a cohort of patients transplanted in the presence of DSA. The aim was to investigate the presence of concurrent donor-specific memory B cell-derived HLA antibodies (DSA-M) and its association with occurrence of AMR.

\section{MATERIALS AND METHODS}

\section{Study Design}

The study (project number FUP092) was conducted within the framework of the Swiss Transplant Cohort Study (STCS), supported by the Swiss National Science Foundation and the Swiss University Hospitals (G15) and transplant centers. ${ }^{19}$ Signed written informed consent was obtained, as approved by the local institutional review board and the STCS committee. We investigated a cohort of DSA-positive patients transplanted at the University Hospital Basel and took advantage of the STCS bio-banking, collecting peripheral blood mononuclear cells (PBMC) and plasma of participating patients at 3 timepoints within the first year: pretransplant, at month $6(\mathrm{~m} 6)$, and at month 12 (m12) after transplantation. Pretransplant samples were either collected on the day of transplantation or maximum 3 days in advance. Biologic material was shipped and all analyses were performed at the Leiden University Medical Center (the Netherlands).

\section{Patient Population}

We identified 64 DSA-positive patients transplanted between May 2008 and December 2015 and participating in the STCS. All patients had pretransplant DSA defined by SAB testing using high resolution HLA typing results but negative $T$ and $B$ cell complement-dependent cytotoxicity crossmatches at the time of transplantation. No flow cytometric crossmatches were performed. In total, we excluded 23 patients for the following reasons: (1) <2 allograft biopsies (indication or surveillance) within the first year posttransplant $(\mathrm{n}=9),(2)$ no induction therapy as intended by the protocol $(\mathrm{n}=5)$, (3) no complete follow-up of at least 12 months $(n=6)$, and (4) lacking or insufficient biologic material at one or more of the investigated timepoints $(n=3)$. For this pilot study, we selected 20 out of the remaining 41 patients with prioritizing those who had higher number of frozen PBMC and distinct histological phenotypes (eg, an AMR phenotype in $\geq 2$ biopsies or no signs of AMR in any biopsy).

\section{Allograft Biopsies and Definition of Rejection}

Indication biopsies were performed at any time in case of inadequate or worsening graft function. According to the local protocol, surveillance biopsies were scheduled at month 3, 6, and 12 posttransplant. Rejection was defined according to the most recent Banff guidelines at the time of study performance. ${ }^{20}$

\section{Immunosuppression}

All patients received induction therapy with a polyclonal T cell-depleting agent (ATG-Fresenius total dose $21 \mathrm{mg} / \mathrm{kg}$ body weight or Thymoglobulin total dose $6 \mathrm{mg} /$ $\mathrm{kg}$ body) plus intravenous immunoglobulins (total dose $2 \mathrm{~g} / \mathrm{kg}$ body weight) and indefinitely continued triple maintenance immunosuppression with tacrolimus, mycophenolic acid, and steroids, as described previously. ${ }^{2,3}$

\section{HLA Typing}

For the purpose of this study, both recipients and donors were retrospectively HLA typed by next generation sequencing for all loci on an Illumina platform (Illumina, San Diego, CA) at the Leiden University Medical Center. Next generation sequencing was performed as previously described. $^{18}$

\section{Polyclonal Activation of B Cells and Supernatant Preparation}

PBMC were isolated using Ficoll-Hypaque density gradient centrifugation and kept frozen in liquid nitrogen until use. Upon thawing, PBMC were polyclonally stimulated for 6-10 days, culture supernatants retrieved, and IgG was isolated and concentrated, as reported before. ${ }^{18}$ Flow cytometry to obtain $\mathrm{CD} 19^{+}$percentages within PBMC was performed before and after stimulation. We determined total IgG concentrations of neat supernatants and eluates by enzyme-linked immunosorbent assay (ELISA), as described previously. ${ }^{18}$

\section{HLA Antibody Detection and DSA Assignment}

Plasma samples and eluates were analyzed for the presence of HLA antibodies using Lifecodes SAB kits (LSA, Immucor Transplant Diagnostics, Stamford, CT). Performance of the assay was based on a previously described protocol. ${ }^{21}$ All plasma samples were treated with ethylene-diamine-tetra-acetat (EDTA). Data analysis was performed using MATCHIT software version 1.3.0 (Immucor). Background corrected mean fluorescence intensity (MFI) values (BCM), BCR (BCM divided by the raw MFI of the lowest ranked bead for a locus), and AD-BCR (antigen density corrected BCR values) were calculated by the software. Beads were assigned as positive if at least 2 of 3 of the following criteria were met: for HLA class I: $\mathrm{BCM}>1000, \mathrm{BCR}>3, \mathrm{AD}-\mathrm{BCR}>4$ and for HLA class II: $\mathrm{BCM}>1000, \mathrm{BCR}>4, \mathrm{AD}-\mathrm{BCR}>5$.

HLA mismatches were defined at the allelic level. DSA were only assigned if the bead of the mismatched allele was present.

\section{Statistical Analyses}

Data were analyzed using JMP Version 13 software (SAS institute Inc., Cary, NC). Categorical data are presented as counts and/or percentages and were analyzed by Fisher's exact test. Continuous data are shown as median and interquartile ranges and compared by Wilcoxon rank sum tests. The Spearman correlation was used as a nonparametric measure of association. Time-to-rejection curves were generated by the Kaplan-Meier method, and the groups compared using the log-rank test. For all statistical tests, a 2 -tailed $P<0.05$ was considered statistically significant. 


\section{RESULTS}

\section{Study Population Characteristics}

In total, 20 kidney transplant recipients were investigated and their characteristics are summarized in Table S1 (SDC, http://links.lww.com/TP/B793). Patients had a median age of 58 years and $45 \%$ were women. Eighty percent of patients received deceased donor transplants. All but 1 patient $(95 \%)$ had a history of known sensitizing events and 13 patients $(65 \%)$ were previously transplanted. Most patients had either 1 or 2 DSA (17/20; $85 \%)$ and 16 of 20 patients $(80 \%)$ class II or class I+II DSA. HLA mismatches and the assigned DSA of all patients are detailed in Table S2 (SDC, http://links.lww. com/TP/B793).

\section{Polyclonal B Cell Stimulation}

Polyclonal activation of PBMC was performed in all patients using samples obtained pretransplant, in 17 of 20 $(85 \%)$ patients at $\mathrm{m} 6$ and in 16 of $20(80 \%)$ patients at $\mathrm{m} 12$. Excluded samples belonged to patients who received rituximab as rejection treatment resulting, as expected, in profound and persisting peripheral $\mathrm{B}$ cell depletion precluding successful polyclonal stimulation of B cells.

Per patient and timepoint, a median number of 16 million PBMC (minimum 8 million) was cultured. The median percentage of $\mathrm{CD} 19^{+} \mathrm{B}$ cells within the lymphocyte gate determined by flow cytometry on day 0 of the culture was $6.3 \%(4.1-13.5)$ pretransplant, 9.9\% (7.4-26.3) at m6, and $10.8 \%(5.7-20.0)$ at $\mathrm{m} 12$. To assure successful polyclonal stimulation, we measured total IgG concentrations by ELISA. Neat culture supernatants showed a median total IgG concentration of $8.8 \mu \mathrm{g} / \mathrm{mL}(4.6-19.8), 12.7 \mu \mathrm{g} /$ $\mathrm{mL}(7.7-27.4)$, and $15.8 \mu \mathrm{g} / \mathrm{mL}(8.1-25.6)$ at the 3 timepoints, respectively, which was within the expected range considering the B cell percentages and our previous experience. ${ }^{18}$ Following IgG isolation and concentration, the median total IgG concentration pretransplant was 1189 $\mu \mathrm{g} / \mathrm{mL}(547-4116)$, and $2240 \mu \mathrm{g} / \mathrm{mL}(1200-4132)$ and $2237 \mu \mathrm{g} / \mathrm{mL}(1574-4641)$ at $\mathrm{m} 6$ and $\mathrm{m} 12$, respectively.

\section{Comparison of HLA Antibody Profiles in Plasma and Eluates}

\section{Pretransplant}

All patients had pretransplant plasma HLA antibodies (DSA and non-DSA). Of those, $80 \%$ had also detectable HLA antibodies deriving from memory B cells (Table S3, SDC, http://links.lww.com/TP/B793). We subsequently focused on donor-directed antibody specificities. In total, the cohort had 35 pretransplant DSA (14 class I, 21 class II). Of those, we detected concurrent DSA-M in 9 patients $(45 \%)$ and for 10 specificities (29\%) (Figure 1A). Of the latter, 4 were class I and 6 class II antibodies. MFI values of DSA with concurrent DSA-M were higher than those of DSA without DSA-M (median 5877 (2441-10 003) and 1476 (943-3901)). Noteworthy, both groups contained DSA with a broad range of MFI values (DSA/DSA-M MFI 783-18 919, DSA/DSA-M ${ }_{\text {neg }}$ : MFI 447-18 808). This was also observed when we investigated class I and II separately. In only 1 patient (patient 9), a class II DSA-M without concurrent DSA was detected.

\section{Posttransplant}

At m6 and m12 posttransplant, only 19 (95\%) and 17 $(85 \%)$ patients still had plasma antibodies (DSA and nonDSA). However, the proportion of patients with detectable memory B cell-derived HLA antibodies remained stable (82\% and $88 \%$, respectively) (Table S3, SDC, http://links. lww.com/TP/B793).

As illustrated in Figure 1A, there were also less donordirected antibody specificities detected posttransplant. At m6, we found 12 DSA in 9 patients and concurrent DSA-M in 2 of $9(22 \%)$ patients and for 2 of $12(17 \%)$ specificities. In 1 patient and for 1 specificity $(A * 32: 01$, patient 6$)$, DSA-M was detected at $\mathrm{m} 6$ while the plasma antibody was not present anymore at this timepoint. At m12, we detected only 7 DSA in 6 patients and 1 patient $(17 \%)$ had concurrent DSA-M.

Based on their persistence or disappearance posttransplant, we then grouped pretransplant DSA of patients who had also posttransplant eluate analyses ( $\mathrm{n}=17$ patients with $n=28$ specificities) into persisting and nonpersisting DSA (Figure 1B). Interestingly, persisting DSA had more frequently DSA-M $(6 / 12 ; 50 \%)$ than nonpersisting DSA $(2 / 16 ; 13 \%)$.

\section{De Novo DSA and DSA-M Development}

We did not detect de novo DSA in any of the 20 study patients at $\mathrm{m} 6$ and $\mathrm{m} 12$ posttransplant. De novo DSA-M without DSA was found in only 1 patient (13) and for 1 specificity ( $A * 31: 01$, data not shown) at $m 6$, but was not anymore detectable at $\mathrm{m} 12$.

\section{Association of Pretransplant DSA-M Status With Transplant Outcomes}

Next, we correlated transplant outcomes with pretransplant presence and absence of DSA-M (Table 1). All patients had a minimal follow-up of 1 year and a median follow-up of 5 years (3.1-7.9).

In total, 73 allograft biopsies were performed. A median number of 4 allograft biopsies was obtained in both DSA$M_{\text {pos }}$ and DSA-M $M_{\text {neg }}$ patients in the first year. Pretransplant DSA-M $M_{\text {pos }}$ patients showed a significantly higher incidence of (sub)clinical AMR $(P=0.032$, Figure 2$)$. In addition, DSA-M $M_{\text {pos }}$ patients had also a higher extent of microvascular inflammation as defined by glomerulitis and peritubular capillaritis scores $\geq 1(67 \%$ versus $9 \%, P=0.02$, Table 1). C4d-positive AMR episodes as well as persistent AMR (defined as an AMR phenotype in at least 2 subsequent biopsies) were more frequently observed in pretransplant DSA-M $M_{\text {pos }}$ patients, but this did not reach statistical significance.

Interestingly, 7 of $9(78 \%)$ patients who had persisting DSA (Figure 1A) developed AMR posttransplant. Of those, DSA-M pretransplant was detected in 5 cases $(5 / 7 ; 71 \%)$.

Three patients lost their graft during the follow-up period. In 2 of 3 patients $(66 \%)$, graft failure, it was due to ongoing humoral rejection (1 DSA-M $\mathrm{M}_{\text {pos }}$ and 1 DSA$M_{\text {neg }}$, respectively). One patient lost the graft following an ischemic event (pneumonia with severe hypotension and stenosis of transplant artery).

Among all patients with a functioning graft at the time of last follow-up, there was a trend towards lower 
A
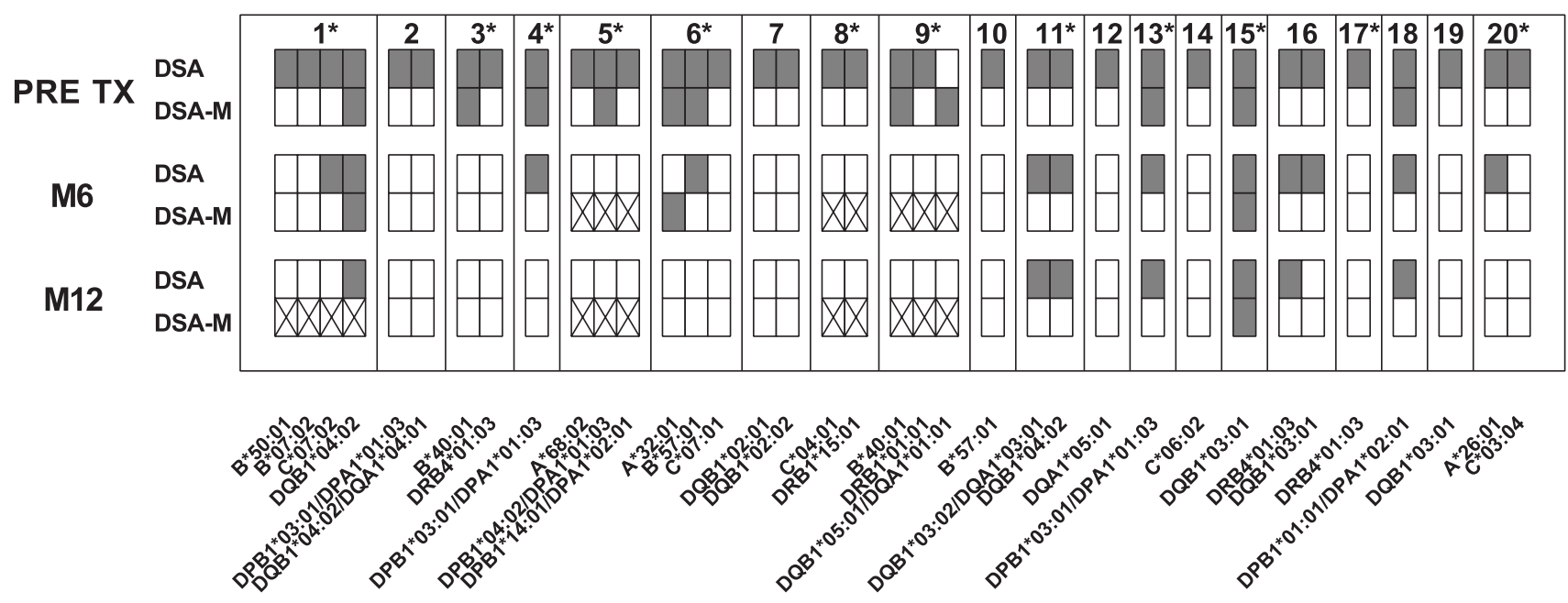

B

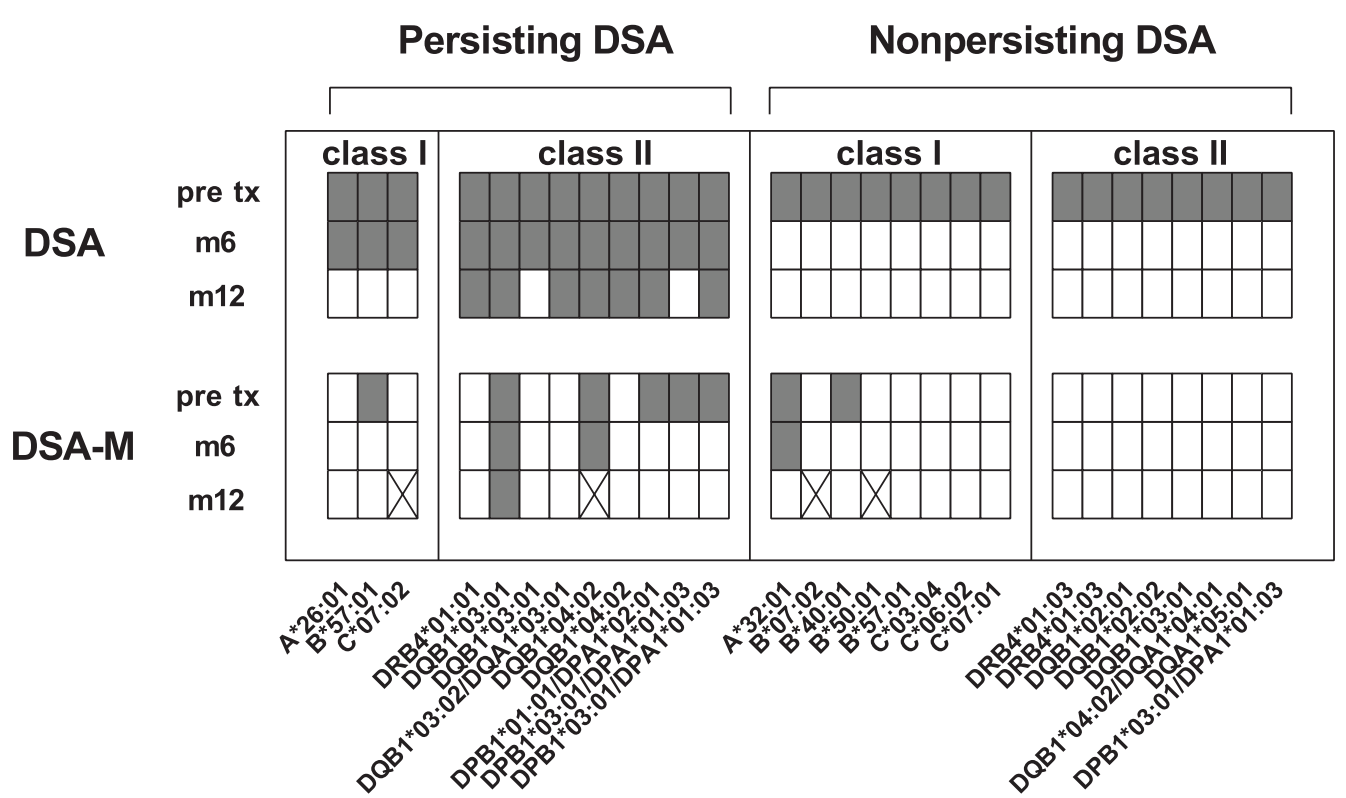

FIGURE 1. Donor-specific HLA antibody profiles in plasma (DSA) and culture supernatants (DSA-M). A, Comparison of DSA and DSA-M before transplantation (pre tx), at mo $6(\mathrm{~m} 6)$, and mo $12(\mathrm{~m} 12)$ in the study population $(\mathrm{n}=20)$. B, Comparison of DSA and DSA-M before transplantation (pre tx), at mo 6 (m6), and mo 12 (m12) in patients with posttransplant eluate analyses ( $\mathrm{n}=17$ patients with $n$ = 28 specificities), grouped according to persistence and HLA class of DSA. Gray-shaded boxes indicate assigned positivity. Boxes marked with $X$ represent samples not tested due to low B cell counts. Patients marked by an asterisk developed AMR posttransplant. AMR, antibody-mediated rejection; DSA, donor-specific HLA antibodies; DSA-M, donor-specific memory B cell-derived HLA antibodies.

estimated glomerular filtration rate in pretransplant DSA$\mathrm{M}_{\text {pos }}$ patients compared with DSA-M $\mathrm{M}_{\text {neg }}$ patients (Table 1).

\section{DISCUSSION}

The main findings of this study are that $45 \%$ of pretransplant DSA-positive patients had detectable concurrent DSA-M and that DSA/DSA-M $M_{\text {oo }}$ individuals more frequently developed (sub)clinical AMR in the first year than DSA/DSA-M $M_{\text {neg }}$ patients. Furthermore, DSA/DSA$M_{\text {pos }}$ patients showed a higher extent of microvascular inflammation in their allograft biopsies, and had a tendency towards worse allograft function at last follow-up. These results are in line with the association between posttransplant detection of circulating HLA-specific donor-directed memory B cells and AMR lesions found in a recently published study using HLA-specific B cell enzyme-linked immunospot assay (ELISPOT) assays. ${ }^{17}$ Importantly, our findings suggest that assessment of the peripheral donor-specific memory B cell pool may be used before transplantation, which is clinically desirable.

An interesting observation was that MFI values of DSA with concurrent DSA-M were higher than those of DSA without DSA-M, although MFI distribution showed a broad range in both groups. On the patient level, pretransplant DSA with MFI $>3000$ were found in $50 \%$ of patients developing AMR and all but 1 had detectable DSA-M (data not shown). Among 6 patients experiencing AMR in the presence of low MFI DSA (MFI <3000, one of them MFI <1000), 3 otherwise unrecognized patients 
TABLE 1.

Association of pretransplant DSA-M status with transplant outcomes

\begin{tabular}{|c|c|c|c|}
\hline & DSA-M $_{\text {pos }}$ pretransplant $(n=9)$ & DSA-M $_{\text {neg }}$ pretransplant $(n=11)$ & $P$-level \\
\hline Total number of biopsies within first y & 35 & 38 & n.a. \\
\hline Median number of biopsies within first y & $4(3-5)$ & $4(3-4)$ & 0.28 \\
\hline \multicolumn{4}{|l|}{ Frequency of rejection in the first y } \\
\hline - Any rejection (subclinical or clinical) & $8(89 \%)$ & $6(55 \%)$ & 0.16 \\
\hline - Any TCMR (subclinical or clinical) & $7(78 \%)$ & $5(45 \%)$ & 0.2 \\
\hline - Any AMR (subclinical or clinical) & $8(89 \%)$ & $4(36 \%)$ & 0.03 \\
\hline \multicolumn{4}{|l|}{ AMR details in the first y } \\
\hline - Clinical AMR & $3(33 \%)$ & $1(9 \%)$ & 0.28 \\
\hline - $g \geq 1$ and $p t c \geq 1$ in $\geq 1$ biopsy & $6(67 \%)$ & $1(9 \%)$ & 0.02 \\
\hline - C4d positive AMR in $\geq 1$ biopsy & $4(44 \%)$ & $3(27 \%)$ & 0.64 \\
\hline - Persistent AMR & $6(67 \%)$ & $4(36 \%)$ & 0.37 \\
\hline Graft failure & $2(22 \%)$ & $1(9 \%)$ & 0.57 \\
\hline Death with functioning graft & $1(11 \%)$ & $2(18 \%)$ & 1.0 \\
\hline \multicolumn{4}{|l|}{ Functioning graft at last follow-up } \\
\hline - creatinine ( $($ mol/l) & $188(135-237)$ & $126(105-175)$ & 0.16 \\
\hline - eGFR MDRD (mL/min) & $32(25-41)$ & $52(28-63)$ & 0.22 \\
\hline - protein/creatinine ratio (mg/mmol) & $19(9-109)$ & $15(7-37)$ & 0.59 \\
\hline
\end{tabular}

AMR, antibody-mediated rejection; DSA-M, donor-specific memory B cell-derived HLA antibodies; eGFR, estimated glomerular filtration rate; g, glomerulitis; MDRD, Modification of Diet in Renal Disease; ptc, peritubular capillaritis; TCMR, T cell-mediated rejection.

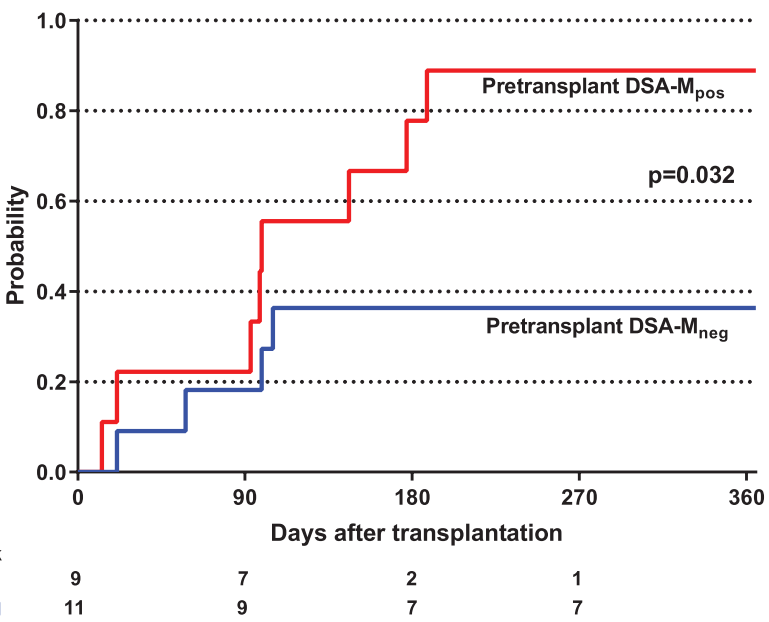

FIGURE 2. Incidence of (sub)clinical AMR in patients with (DSA$M_{\text {pos }}$ ) and without (DSA-M $M_{\text {neg }}$ ) pretransplant memory B cell-derived donor-specific HLA antibodies. AMR, antibody-mediated rejection; DSA-M, donor-specific memory B cell-derived HLA antibodies.

would have been identified as being at higher risk using the new method. Supported by these findings, use of an integrative approach with assessment of multiple factors being involved in the alloimmune response is advisable. ${ }^{22-24}$

Mechanistically, HLA-specific memory B cells can contribute to the antibody repertoire by differentiating into antibody-producing cells following antigen rechallenge, thereby serving as a replenishing source of circulating DSA. ${ }^{25}$ Not surprisingly, we observed that persisting posttransplant DSA were more often found to have DSA-M than those becoming undetectable. In the majority (7/9; $78 \%$ ) of patients with persisting DSA, AMR was diagnosed within the first year. Future studies should also address the question whether detection of pretransplant DSA-M predicts the persistence of DSA, since this may abolish the need to monitor the evolution of preexisting antibodies after transplantation.
Several studies have shown that HLA antibody profiles from serum and culture supernatants overlap but are not mirror images of each other. ${ }^{14,16,26,27}$ An important clinical question is whether DSA-M can be detected while plasma antibodies are absent. In this study, we detected only 1 DSA-M that was not present in the plasma, which might be due to the study design focusing on DSA-positive patients. Likely, the frequency of detecting DSA-M without DSA will be considerably higher in specific clinical situations such as husband-to-wife (with shared children) or child-to-mother transplantation.

Absence of de novo DSA and only transient de novo DSA-M formation for 1 specificity was observed in this study. Noteworthy, lacking detection of de novo DSA up to 1 year posttransplant is not surprising as the frequency of de novo DSA in the first year has been found to be around $2 \%{ }^{28,29}$ In addition, $\mathrm{T}$ cell-depleting therapy with ATG has recently been shown to dampen both naive and memory DSA responses by impaired $\mathrm{CD}^{+} \mathrm{T}$ cell help and can therefore be considered as an effective prevention of de novo DSA development until T-cell recovery. ${ }^{30}$

This study has several strengths. Assignment of DSA was performed in the most accurate way, namely by combination of high resolution HLA typing results of all loci of donors and recipients and antibody detection using SAB technology. All patients received uniform induction therapy and maintenance immunosuppression. In addition, all transplant recipients underwent surveillance biopsies and had a median number of 4 allograft biopsies per patient, making it rather unlikely that AMR episodes within the first year were missed.

The main limitation is the small number of patients, which is inherent to the pilot character of this study and only allows the detection of a possible association with the occurrence of AMR. Since we focused on a specific subgroup of patients transplanted in the presence of DSA following $\mathrm{T}$ cell-depleting therapy and retrospectively selected patients based on availability of biological material as well 
as histological phenotypes, a selection bias cannot be ruled out. Methodologically, antibodies contained in culture supernatants are only qualitatively analyzed. Quantification of memory B cells is, however, currently only possible by performing labor-intensive and less sensitive ELISPOT assays. ${ }^{15,16}$ In addition, due to circulation between the secondary lymphoid organs and the peripheral blood, memory $\mathrm{B}$ cells may appear in the latter in low frequencies, which potentially precludes their detection. This limitation applies to both ELISPOT assays and culture supernatant analyses. For clinical purposes, it is therefore important to focus on their detectability instead of their absence, which is the approach we followed in this study.

In conclusion, the current data suggest that assessment of DSA-M provides a new tool that could supplement serum HLA antibody analysis for pretransplant risk stratification in DSA-positive patients. Validation of our findings in a larger cohort and expansion to other patient groups, such as patients without pretransplant DSA, is warranted.

\section{ACKNOWLEDGMENTS}

The authors thank Jos Drabbels and Pieter van der Linden for technical assistance in HLA typing by NGS. Furthermore, the authors wish to thank Aynur Gubelmann, Joëlle Handschin, Gideon Hönger, and Claudia Petit of the HLA laboratory in Basel (Switzerland) for preparation of shipping of the patient cells and DNA samples to Leiden (the Netherlands). This study has been conducted in the framework of the Swiss Transplant Cohort Study, supported by the Swiss National Science Foundation and the Swiss University Hospitals (G15) and transplant centers.

*The members of the Swiss Transplant Cohort Study: Patrizia Amico, John-David Aubert, Vanessa Banz, Guido Beldi, Christian Benden, Christoph Berger, Isabelle Binet, Pierre-Yves Bochud, Elsa Boëly, Sanda Branca, Heiner Bucher, Thierry Carell, Emmanuelle Catana, Yves Chalandon, Sabina de Geest, Olivier de Rougemont, Michael Dickenmann, Michel Duchosal, Laure Elkrief, Thomas Fehr, Sylvie Ferrari-Lacraz, Christian Garzoni, Paola Gasche Soccal, Christophe Gaudet, Emiliano Giostra, Déla Golshayan, Karine Hadaya, Jörg Halter, Dominik Heim, Christoph Hess, Sven Hillinger, Hans H. Hirsch, Günther Hofbauer, Uyen Huynh-Do, Franz Immer, Richard Klaghofer, Michael Koller (Head of the data center), Bettina Laesser, Roger Lehmann, Christian Lovis, Pietro Majno; Oriol Manuel, Hans-Peter Marti, Pierre Yves Martin, Pascal Meylan, (Head, Biological samples management group), Paul Mohacsi, Philippe Morel, Ulrike Mueller, Nicolas J. Mueller (Chairman Scientific Committee), Helen Mueller-McKenna (Head of local data management), Antonia Müller, Thomas Müller, Beat Müllhaupt, Manuel Pascual (Executive office), Jakob Passweg, Klara Posfay-Barbe, Juliane Rick, Eddy Roosnek, Anne Rosselet, Silvia Rothlin, Frank Ruschitzka, Urs Schanz, Stefan Schaub, Aurelia Schnyder, Christian Seiler, Jan Sprachta; Susanne Stampf, Jürg Steiger (Head, Executive Office), Guido Stirnimann, Christian Toso, Christian Van Delden (Executive office), Jean-Pierre Venetz, Jean Villard, Madeleine Wick (STCS coordinator), Markus Wilhelm, and Patrick Yerly.

\section{REFERENCES}

1. Lefaucheur C, Loupy A, Hill GS, et al. Preexisting donor-specific HLA antibodies predict outcome in kidney transplantation. J Am Soc Nephrol. 2010;21:1398-1406.

2. Wehmeier $\mathrm{C}$, Hönger $\mathrm{G}$, Cun $\mathrm{H}$, et al. Donor specificity but not broadness of sensitization is associated with antibody-mediated rejection and graft loss in renal allograft recipients. Am J Transplant. 2017;17:2092-2102

3. Bächler K, Amico P, Hönger G, et al. Efficacy of induction therapy with ATG and intravenous immunoglobulins in patients with low-level donorspecific HLA-antibodies. Am J Transplant. 2010;10:1254-1262.

4. Marfo K, Ajaimy M, Colovai A, et al. Pretransplant immunologic risk assessment of kidney transplant recipients with donor-specific anti-human leukocyte antigen antibodies. Transplantation. 2014;98:1082-1088

5. Montgomery RA, Lonze BE, King KE, et al. Desensitization in HLA-incompatible kidney recipients and survival. N Engl J Med. 2011;365:318-326.

6. Courant M, Visentin J, Linares $G$, et al. The disappointing contribution of anti-human leukocyte antigen donor-specific antibodies characteristics for predicting allograft loss. Nephrol Dial Transplant. 2018;33:1853-1863.

7. Valenzuela NM, Schaub S. The biology of IgG subclasses and their clinical relevance to transplantation. Transplantation. 2018;102(1S Suppl 1):S7-S13.

8. Burns JM, Cornell LD, Perry DK, et al. Alloantibody levels and acute humoral rejection early after positive crossmatch kidney transplantation. Am J Transplant. 2008;8:2684-2694.

9. Kimball PM, Baker MA, Wagner MB, et al. Surveillance of alloantibodies after transplantation identifies the risk of chronic rejection. Kidney Int. 2011;79:1131-1137.

10. Redondo-Pachón D, Pérez-Sáez MJ, Mir M, et al. Impact of persistent and cleared preformed HLA DSA on kidney transplant outcomes. Hum Immunol. 2018;79:424-431.

11. Bernasconi NL, Traggiai E, Lanzavecchia A. Maintenance of serological memory by polyclonal activation of human memory B cells. Science. 2002;298(5601):2199-2202.

12. Crotty S, Ahmed R. Immunological memory in humans. Semin Immunol. 2004;16:197-203.

13. D'Orsogna $L$, van den Heuvel $H$, van Kooten $C$, et al. Infectious pathogens may trigger specific allo-HLA reactivity via multiple mechanisms. Immunogenetics. 2017;69(8-9):631-641.

14. Han M, Rogers JA, Lavingia B, et al. Peripheral blood B cells producing donor-specific HLA antibodies in vitro. Hum Immunol. 2009;70:29-34.

15. Karahan GE, de Vaal YJH, Krop J, et al. A memory B cell crossmatch assay for quantification of donor-specific memory $B$ cells in the peripheral blood of HLA-immunized individuals. Am J Transplant. 2017; 17:2617-2626

16. Lúcia M, Luque S, Crespo E, et al. Preformed circulating HLA-specific memory B cells predict high risk of humoral rejection in kidney transplantation. Kidney Int. 2015;88:874-887.

17. Luque $S$, Lúcia M, Melilli E, et al. Value of monitoring circulating donorreactive memory $\mathrm{B}$ cells to characterize antibody-mediated rejection after kidney transplantation. Am J Transplant. 2019;19:368-380.

18. Karahan GE, Krop J, Wehmeier C, et al. An easy and sensitive method to profile the antibody specificities of HLA-specific memory B cells. Transplantation. 2019;103:716-723.

19. Yamakuchi M, Kirkiles-Smith NC, Ferlito M, et al. Antibody to human leukocyte antigen triggers endothelial exocytosis. Proc Natl $\mathrm{Acad} \mathrm{Scl}$ U S A. 2007;104:1301-1306.

20. Loupy A, Haas M, Solez K, et al. The Banff 2015 kidney meeting report: current challenges in rejection classification and prospects for adopting molecular pathology. Am J Transplant. 2017;17:28-41.

21. Kamburova EG, Wisse BW, Joosten I, et al. How can we reduce costs of solid-phase multiplex-bead assays used to determine anti-HLA antibodies? HLA. 2016;88:110-119.

22. Lefaucheur C, Viglietti D, Mangiola M, et al. From humoral theory to performant risk stratification in kidney transplantation. J Immunol Res. 2017;2017:5201098.

23. Tambur AR, Campbell P, Claas FH, et al. Sensitization in Transplantation: Assessment of Risk (STAR) 2017 working group meeting report. Am J Transplant. 2018;18:1604-1614.

24. Wiebe C, Ho J, Gibson IW, et al. Carpe diem-time to transition from empiric to precision medicine in kidney transplantation. $\mathrm{Am} \mathrm{J}$ Transplant. 2018;18:1615-1625. 
25. Chong AS, Ansari MJ. Heterogeneity of memory B cells. Am J Transplant. 2018;18:779-784.

26. Snanoudj R, Claas FH, Heidt S, et al. Restricted specificity of peripheral alloreactive memory B cells in HLA-sensitized patients awaiting a kidney transplant. Kidney Int. 2015;87:1230-1240.

27. Karahan GE, Claas FH, Heidt S. Detecting the humoral alloimmune response: we need more than serum antibody screening. Transplantation. 2015;99:908-915.
28. Wehmeier $\mathrm{C}$, Amico P, Hirt-Minkowski P, et al. Acute rejection phenotypes in the current era of immunosuppression: a single-center analysis. Transplant Direct. 2017;3:e136.

29. Wiebe C, Gibson IW, Blydt-Hansen TD, et al. Rates and determinants of progression to graft failure in kidney allograft recipients with de novo donor-specific antibody. Am J Transplant. 2015;15:2921-2930.

30. Chen CC, Koenig A, Saison C, et al. CD4+ T cell help is mandatory for naive and memory donor-specific antibody responses: impact of therapeutic immunosuppression. Front Immunol. 2018;9:275. 